\title{
OZI Rule and Instantons
}

\author{
M.A. Nowak ${ }^{1}$ \\ Physics Department, SUNY \\ Stony Brook, New York, 11794, USA \\ J.J.M. Verbaarschot \\ CERN Theory Division \\ CH1211 Geneva 23, Switzerland \\ I. Zahed \\ Physics Department, SUNY \\ Stony Brook, New York, 11794, USA
}

\begin{abstract}
We investigate the effects of strangeness mixing in the QCD vacuum described as a quantum disordered phase of instantons and anti-instantons. We find that the constituent mass of the light quarks is a decreasing function of the current quark masses, and that its mass is almost insensitive to the value of the strange quark mass. Our results cannot account for a breach in the OZI rule in the vacuum. The relevance of this result for the amount of $s \bar{s}$ pairs in the nucleon and the pion-nucleon sigma term is discussed.
\end{abstract}

${ }^{1}$ On leave of absence from The Institute of Physics, Jagellonian University, Cracow, Poland.

CERN-TH-5387/89

May 1989 
The issue of flavor mixing in QCD at low energy depends fundamentally on our understanding of the QCD vacuum. The latter is known to contain non-trivial gauge configurations responsible for the formation of a quark and gluon condensate. What is less known is whether these configurations are classical gauge configurations such as instantons or monopoles, or purely quantum gluon fluctuations. There is some qualitative evidence, mainly from the QCD sum rules [1], in favor of the quasi-classical approach.

In so far, there have been various attempts to describe the $\mathrm{QCD}$ vacuum in terms of classical gauge configurations dressed with small quantum fluctuations. The instanton approach to the QCD vacuum, while it does not comply with the general requirement of confinement at large distance scales, seems to capture the essence of chiral symmetry breaking at short distance scales $[2,3,4,5]$. Due to quantum disorder, instantons and anti-instantons in the vacuum stabilize in a dilute liquid state, where light quarks are delocalized over a range of the order of their constituent mass. The latter is dynamically generated in the liquid state (see e.g. refs. $[6,7,8,9])$.

In a recent work $[9,10]$ we investigated the dependence of the chiral condensate on the strange current quark mass for a fixed average density and size of the instantons in the vacuum. We concluded that the results of this model are in agreement with the OZI rule and a priori inconsistent with a large pion-nucleon sigma term $[11,12]$. The purpose of this letter is to further investigate the effects of flavor mixing in general, and strangeness mixing in particular. We will study the sensitivity of the average density and the size of the instantons in the vacuum on the current quark masses and evaluate the effects (if any) on the constituent quark mass. The phenomenological relevance of our results will be discussed.

The vacuum viewed as a dilute liquid of instantons and anti-instantons is topologically neutral. The grand partition function of a neutral system of $N_{+}$instantons and $N_{-}$anti-instantons can be approximated by $[13,14,6]$ 


$$
Z=\sum_{N_{+}, N_{\sim}} \frac{1}{N_{+} ! N_{-} !} \int \prod_{I}^{N} d^{4} z_{I} d U_{I} d \rho_{I} d\left(\rho_{I}\right) \exp \left(-\beta(\bar{\rho}) u_{i n t}\right) \operatorname{det}\left(-i \gamma_{\mu} \nabla_{\mu}-i m\right)
$$

where the integration is over the collective variables denoting the position $\left(z_{I}\right)$ in four-dimensional Euclidean space, the color orientation $\left(U_{I}\right)$ in $S U\left(N_{c}\right)$ and the size $\left(\rho_{I}\right)$ of the pseudoparticles. The size distribution $d(\rho)$ is given by

$$
d(\rho)=C \rho^{-5}\left(\frac{8 \pi^{2}}{g^{2}}\right)^{2 N_{c}}(M \rho)^{\frac{2}{3} N_{f}} \exp -\beta(\rho),
$$

where $\beta$ is the one-loop Gell-Mann Low function evaluated at a scale that is of the order of the size $\rho$ of the instantons

$$
\beta(\rho)=-b \log (\rho \Lambda), \quad b=\frac{11}{3} N_{c}-\frac{2}{3} N_{f}
$$

and $C=4.60 \exp \left(-1.68 N_{c}\right) /\left(\pi^{2}\left(N_{c}-1\right) !\left(N_{c}-2\right) !\right) . \Lambda$ is the ultra-violet cut-off in the Pauli-Villars scheme, and $M$ is a renormalization mass. The argument of the beta function in (1) is the average size $\bar{\rho}$ of the instantons. In (1) $u_{\text {int }}$ is short for the two-body interaction between an instanton and an anti-instanton. In general, it depends on their respective sizes, color orientations and separations. On the average, it is repulsive [15].

The fermion determinant in (1) involves the covariant derivative $\nabla$ in the classical background of instantons and anti-instantons (pseudoparticles), and a diagonal mass matrix $m=\operatorname{diag}\left(m_{1}, \ldots, m_{f}\right)$ for $N_{f}$ flavors. It can be factorized in a low momentum factor $\left(\equiv \operatorname{det}_{\text {low }}\right)$ and a high momentum factor $\left(\equiv \operatorname{det}_{\text {high }}\right)$. Both factors are regularized by a mass $M$ and normalized by the non-interacting fermion determinant [6]. Specifically,

$$
\operatorname{det}_{h i g h}=\prod_{I, f}\left(M \rho_{I}\right)^{-\frac{2}{3}} 1.339 M_{1} \rho_{I}, \quad M_{1} \rho_{I} \ll 1,
$$


where $M_{1}$ is an arbitrary mass scale separating the low momentum components in the fermion determinant from the high momentum ones. The low momentum part of the fermion determinant can be saturated by the fermionic zero modes [6]

$$
\operatorname{det}_{l o w}=\prod_{f} \operatorname{det}\left(T+i m_{f} K-i m_{f}\right) / \operatorname{det}\left(T+i m_{f} K-i M_{1}\right),
$$

where the matrix elements of the hermitean matrices $T$ and $K$ are defined as follows

$$
\begin{aligned}
T_{I J} & =\int d^{4} x \phi_{I}^{\dagger} i \gamma_{\mu} \partial_{\mu} \phi_{J} \\
K_{I J} & =\int d^{4} x \phi_{I}^{\dagger} \phi_{J}-\delta_{I J}
\end{aligned}
$$

Note that the determinants in eq. (5) are real. $\phi_{I}$ represents the fermionic zero mode in the field of one-instanton or one-anti-instanton. The consistency of this approach requires that the final results are independent of $M_{1}$, i.e. independent of the separation into low and high momenta. This point will be verified below.

We will study the limit in which the overlap matrix $K$ can be neglected. This corresponds to the limit of small current quark masses and a dilute instanton liquid [6]. In this case the distribution of the eigenvalues of the overlap matrix $T$ is semicircular [6] to leading order in $N_{c}$. This greatly simplifies the evaluation of the fermion determinant. In the thermodynamic limit $\left(N / V_{4}=\right.$ constant, $N \rightarrow$ $\left.\infty, V_{4} \rightarrow \infty\right)$ the average of the determinant in (5) can be approximated by a leading order cumulant expansion. The result is

$$
<\log \operatorname{det}\left(T T^{\dagger}+m^{2}\right)>=\frac{N}{\pi \kappa} \int_{-2 \kappa}^{2 \kappa} d \lambda\left(1-\frac{\lambda^{2}}{4 \kappa^{2}}\right)^{\frac{1}{2}} \log \left(m^{2}+\lambda^{2}\right)
$$

where $\langle$.$\rangle is short for averaging over the distribution of the collective coordi-$ nates. For a large number of colors $N_{c}$, the variance $\kappa$ of the eigenvalues of $T$ can be evaluated using a mean field approximation [16] 


$$
\kappa^{2}=6.62 \frac{N}{V_{4} N_{c}} \overline{\rho^{2}}
$$

Performing the integral over the eigenvalue distribution in (7) and collecting all other factors yield the following result for the full fermion determinant,

$$
\prod_{I}\left(1.34 M_{1} \rho_{I}\right)^{N_{f}}\left(\rho_{I} M\right)^{-\frac{2}{3} N_{f}} \prod_{f} H\left(m_{f}\right) / H\left(M_{1}\right)
$$

where

$$
H(m)=\left(\frac{m}{2}+\frac{1}{2}\left(m^{2}+4 \kappa^{2}\right)^{\frac{1}{2}}\right) \exp \frac{1}{2} \frac{m-\left(m^{2}+4 \kappa^{2}\right)^{\frac{1}{2}}}{m+\left(m^{2}+4 \kappa^{2}\right)^{\frac{1}{2}}} .
$$

Notice that in the limit $1 / \vec{\rho} \gg M_{1} \gg \kappa$ the $M_{1}$ dependence in eq (10) cancels and the result is independent of the splitting into low and high momenta. In the low-density limit, this result reproduces the mass dependence originally found by 't Hooft [13].

For large values of the current quark mass we have to take into account the effects due to the overlap matrix $K$. Also the mixing of the zero modes with the continuum states becomes important. Technically, we are unable to handle this mixing. However, $H(m)$ in (10) evaluated for small current quark masses has the correct $m$-dependence for large $m$. Therefore we expect eq. (10) to be a good interpolating formula for all values of the quark masses.

Another argument in favor of eq. (10) is obtained by noting that to lowest order in the current quark masses $H(m)$ is given by

$$
H(m) \rightarrow m+\kappa, \quad m \rightarrow 0
$$

This result is qualitativaly similar to the result advocated by Shifman et al. [17] using the operator product expansion. Their result can be simply obtained in our framework by evaluating the non-perturbative part of the fermion determinant 
using a zero mode approximation [9]. In this approach, the current mass in the size distribution of the instantons is replaced by a determinantal mass $M_{\Delta}$

$$
M_{\Delta}=m+\frac{i}{2 N_{c}} \int \frac{d^{4} k}{(2 \pi)^{4}} k^{2} \phi^{2}(k)<\psi_{\alpha}^{\dagger}(k) \psi_{\alpha}(-k)>.
$$

Here the vacuum expectation value is denoted by $\langle\cdot\rangle$, and the function $\phi^{\prime}(k)$ is related to the Fourier transform of the fermionic zero mode in the one-instanton background [6]. At low momentum $(k \rightarrow 0)$ this function approaches $-2 \pi \rho /|k|$. Replacing $\phi^{\prime}(k)$ by this limit reproduces the result of Shifman et al. [17],

$$
M_{\Delta}=m+\frac{2 \pi^{2} \rho^{2}}{N_{c}} i<\psi^{\dagger} \psi>
$$

where $\left\langle\psi^{\dagger} \psi\right\rangle$ is the Euclidean chiral condensate.

In order to see the connection between eqs. (12) and (14), we have to evaluate the momentum integral in eq. (14). This can be achieved by using the explicit expression for the the quark propagator in the instanton medium,

$$
<\psi_{\alpha}(k) \psi_{\beta}^{\dagger}(-k)>=\left(\gamma_{\mu} k_{\mu}-i M(k)\right)_{\alpha \beta}^{-1}
$$

Notice that the fermion propagator is diagonal in flavor space. Here $M(k)$ is a momentum dependent mass ("constituent mass") not to be confused with the determinantal mass $M_{\Delta}$. The mass $M(k)$ is given by

$$
M(k)=\frac{\epsilon N}{2 V N_{c}} k^{2} \phi^{2}(k)
$$

and satisfies the following gap equation

$$
\int \frac{d^{4} k}{(2 \pi)^{4}} \frac{M^{2}(k)}{k^{2}+M^{2}(k)}=\frac{N}{4 V_{4} N_{c}}(1-m \epsilon) .
$$

Using (16-18), the determinantal quark mass $M_{\Delta}$ is given by

$$
M_{\Delta}=\frac{m}{2}+\frac{1}{2}\left(m^{2}+4 \kappa^{2}\right)^{\frac{1}{2}}
$$


which is exactly the pre-exponential factor given in eq. (11). The determinantal mass obtained in this way $(\approx 80 \mathrm{MeV})$ is roughly a factor 3 smaller than the zero momentum determinantal mass obtained by Shifman et al. [17]. This will very likely modify their estimates on the range of validity of the instanton liquid model.

The average size and density of the instantons in the presence of the fermion determinant is evaluated according to a procedure proposed by Dyakonov and Petrov [15]. They estimate the two-body interaction $u_{\text {int }}$ between instantons in (1) by using the Feynman variational principle under the assumption that the background field is given by a linear superposition of one-instanton and one-anti-instanton configurations. In this way the two-body interaction in (1) can be taken into account in terms of an effective one-body distribution $\mu(\rho)$. In the variational approach the interaction enters only as an average over the collective coordinates (denoted by a bar) leading to a considerable simplification of the analysis. Specifically,

$$
\overline{u_{\text {int }}}=\gamma^{2} \overline{\rho_{1}^{2} \rho_{2}^{2}}, \quad \gamma^{2}=\frac{27 \pi^{2}}{4} \frac{N_{c}}{N_{c}^{2}-1}
$$

The solution of the variational equation for $\mu$ is [18]

$$
\mu(\rho)=d(\rho) \exp \left(\frac{1}{2} \sum_{f} \frac{1}{H}\left(m_{f}\right) \frac{\partial H\left(m_{f}\right)}{\partial \bar{\rho}}(\rho-\bar{\rho})-\beta(\bar{\rho}) \gamma^{2} n \overline{\rho^{2}}\left(\rho^{2}-\overline{\rho^{2}}\right)\right)
$$

where $d$ has been defined in (2) and involves a factor originating from the fermion determinant. The average squared size is determined self-consistently by

$$
\overline{\rho^{2}}=\int d \rho \rho^{2} \mu(\rho) / \int d \rho \mu(\rho),
$$

and $\bar{\rho}$ is defined by $\left(\overrightarrow{\rho^{2}}\right)^{\frac{1}{2}}$. The density $N / V_{4}$ of pseudoparticles in the vacuum is denoted by $n$. In terms of $\mu$ the free energy defined as $F \equiv \log Z$ assumes a particularly simple form 


$$
F=N\left[-\log \frac{n}{2 \Lambda^{4}}+\log \int d \rho \mu(\rho) \Lambda^{4} \exp \left(-\frac{1}{2} \beta(\bar{\rho}) \gamma^{2} n{\overline{\rho^{2}}}^{2}\right)\right]
$$

By maximizing the free energy with respect to $N_{+}$and $N_{-}$we find the equilibrium density of the pseudoparticles. It is clear from (22) that for zero $\theta$ angle we have $N_{+}=N_{-}$. For zero masses the variance of the total number of pseudoparticles is equal to $4 / b \bar{n}[6]$ in agreement with the low-energy theorems derived by Novikov et al. $[19,20]$. Notice that the variance in the number of pseudoparticles in the vacuum is stable in the large $N_{c}$ limit.

In general the average size $\bar{\rho}$ and density $\bar{n}$ of the pseudoparticles have to be determined numerically. In Figs. 1 and 2 we show their dependence on the strange mass in the case of three flavors. The mass of the light $(u, d)$ quarks is $0.024 \Lambda$. We find that the average density of the pseudoparticles increases as a function of $m_{s}$, whereas their average size decreases fast enough so that the product $n \overline{\rho^{4}}$ decreases a function of $m_{s}$. Since at the one loop level the coupling constant $8 \pi^{2} / g^{2}$ is not renormalized we resolve this ambiguity by choosing its value equal to $-b \log (0.1)$. (The same approach was followed in ref. [21].) With a cut-off $\Lambda$ of $250 \mathrm{MeV}$ this gives rise to phenomenologically acceptable numbers. More specifically, for the values of the three current quark masses equal to $6 \mathrm{MeV}, 6 \mathrm{MeV}$ and $150 \mathrm{MeV}$, we find $(190 \mathrm{MeV})^{4}$ for the gluon condensate. The width of the eigenvalues is $\kappa / \Lambda=0.7$ and the size of the pseudoparticles is $\bar{\rho} \Lambda=0.45$, making a separation between low and high momenta possible. The reader can verify that our system is dilute and that the use of a semi-classical description is justified.

Now, we are ready to evaluate the sensitivity of the mass $M_{u}$ (defined in eqs. (16) and (17); the subscript denotes the flavor) to variations of the current quark masses in the instanton vacuum, in particular, to variations of the strange quark mass. For that, we need the solution of the gap equation (17). This equation depends on the dimensionless parameters $\bar{n} \overline{\rho^{4}}$ and $m_{f} \bar{\rho}$. Notice however, that at the mean field level there is no coupling between the various flavors other than 
through the implicit quark mass dependence of the vacuum parameters (density and size of the instantons). This explains why the numerical values of -0.11 and -0.34 we find for $\partial_{m_{s}} M_{u}$ and $\partial_{m_{d}} M_{u}$, respectively, are an order of magnitude smaller than the numerical value of -2.7 we find for $\partial_{m_{u}} M_{u}$. The most surprising feature of these results is their sign. In particular, the sign of $\partial_{m_{u}} M_{u}$ will not be altered by taking into account corrections due to the finite quarks masses. To lowest order the constituent mass will be corrected by the value of the current mass, adding 1 to the derivative. If we assume that the nucleon mass is given by the sum of three consituent masses, this result leads to a negative value for $\Sigma_{\pi N}$, in disagreement with the experiment. In a more realistic model of the nucleon the constituent masses are bound. Taking into account the momentum dependence of $M(k)$ we obtain from eqs. (16-18) a constituent mass of $\approx 500 \mathrm{MeV}$. In order to describe the nucleon in our model we need a binding energy of $\approx 500 \mathrm{MeV}$. Apparently, the current quark mass dependence of the binding energy is important. However, in the present framework we are not able to estimate this effect. Our main conclusion is that the constituent mass is nearly insentive to the strange quark mass. This result is to be contrasted with results from the chiral bag model [22], the Skyrme model result [12], and the NJL result of [23]. In particular, the first two models give rise to a large strangeness admixture in the nucleon state.

The determinantal mass $M_{\Delta}$ shows a different dependence on the current quark mass. First, the sign of $M_{\Delta}$ with respect to the current quark mass is positive. Second, the values of the derivatives are smaller. For the derivatives of $M_{\Delta u}$ with respect to $m_{u}, m_{d}$ and $m_{s}$ we find $0.54,0.028$ and 0.022 , respectively. However, the value of $M_{\Delta u}$ is only $80 \mathrm{MeV}$.

Our results were obtained under the assumption that the collective coordinates of the pseudoparticles are distributed according to their invariant measure. Actually, their distribution should be weighted by the fermion determinant thereby introducing an additional mass dependence. Numerical simulation of the fermion determinant for fixed average size and average density were carried out in refs. 
$[8,10,9]$. The results provided evidence for the hypothesis that in the thermodynamic limit the chiral condensate is insensitive to variations of the quark masses.

In conclusion, we find that the mass dependence of the average size and density of the instantons gives rise to some mixing between quarks of different flavors in the $\mathrm{QCD}$ vacuum described as a liquid of instantons and anti-instantons. The mixing is an order of magnitude less than the one advocated by the Skyrme model. However, the negative value of the pion-nucleon $\Sigma$ term indicates that the binding energy of the constituent quarks has a strong mass dependence which we hope to study in a future publication. Over all, our results indicate that there is no strong violation of the OZI rule in the instanton vacuum.

\section{Acknowledgements}

This work was supported in part by the U.S. Department of Energy under Contract No. DE-FG02-88ER40388. We would like to thank E.V. Shuryak and E.M. Ilgenfritz for useful discussions. 


\section{References}

[1] M.A. Shifman, A.I. Vainshtein, and V.I. Zakharov, Nucl. Phys., B147 (1979)385,448,519.

[2] E.V. Shuryak, Phys. Lett., B203 (1982)93.

[3] E.V. Shuryak, Nucl. Phys., B203 (1982)116.

[4] E.V. Shuryak, Nucl. Phys., B203 (1982)140.

[5] E.V. Shuryak, Nucl. Phys., B214 (1983)237.

[6] D.I. Dyakonov and V.Yu. Petrov, Nucl. Phys., B272 (1986)457.

[7] E.V. Shuryak, Nucl. Phys., B302 (1988)559.

[8] E.V. Shuryak, Nucl. Phys., B302 (1988)599.

[9] M.A. Nowak, J.J.M. Verbaarschot, and I. Zahed, Stony Brook preprint, July 1988, Nucl.Phys.B (in press).

[10] M.A. Nowak, J.J.M. Verbaarschot, and I. Zahed, Phys. Lett., 217B (1989)157.

[11] R.L. Jaffe and C.L. Korpa, Comm. Nucl. Part. Phys., 17 (1987)163.

[12] J.F. Donoghue and C.R. Nappi, Phys. Lett., $168 B$ (1986)105.

[13] G. 't Hooft, Phys.Rev., D14 (1976)3432.

[14] C.G. Callan, R.F. Dashen, and D.J. Gross, Phys.Rev, D17 (1978)2717.

[15] D.I. Dyakonov and V.Yu. Petrov, Nucl. Phys., B245 (1984)259.

[16] D.I. Dyakonov and V.Yu. Petrov, JETP, 89 (1985)361.

[17] M.A. Shifman, A.I. Vainshtein, and V.I. Zakharov, Nucl.Phys., B163 (1980)43. 
[18] M.A. Nowak, J.J.M. Verbaarschot, and I. Zahed, CERN Preprint H-5275/89 (1989) Nucl. Phys. B (in press).

[19] V.A. Novikov, M.A. Shifman, A.I. Vainshtein, and V.I. Zakharov, Nucl.Phys., B191 (1981)301.

[20] V.A. Novikov, M.A. Shifman, A.I. Vainshtein, and V.I. Zakharov, Nucl.Phys., B165 (1980)67.

[21] E.M. Ilgenfritz and E.V. Shuryak, Novosibirsk preprint 88-85, 1988.

[22] G.E. Brown, K. Kubodera, and M. Rho, Phys. Lett., 192B (1987)273.

[23] V. Bernard, R.L. Jaffe, and U.G. Meissner, MIT preprint CTP-1547, 1988. 


\section{Figure Captions}

Fig. [1]. The $m_{s} \Lambda$ dependence of the average density $n \Lambda^{4}$ of the pseudoparticles.

Fig. [2]. The dimensionless average size $\bar{\rho} \Lambda$ as a function of the strange mass $m_{s}$ in units of the cut-off $\Lambda$. 


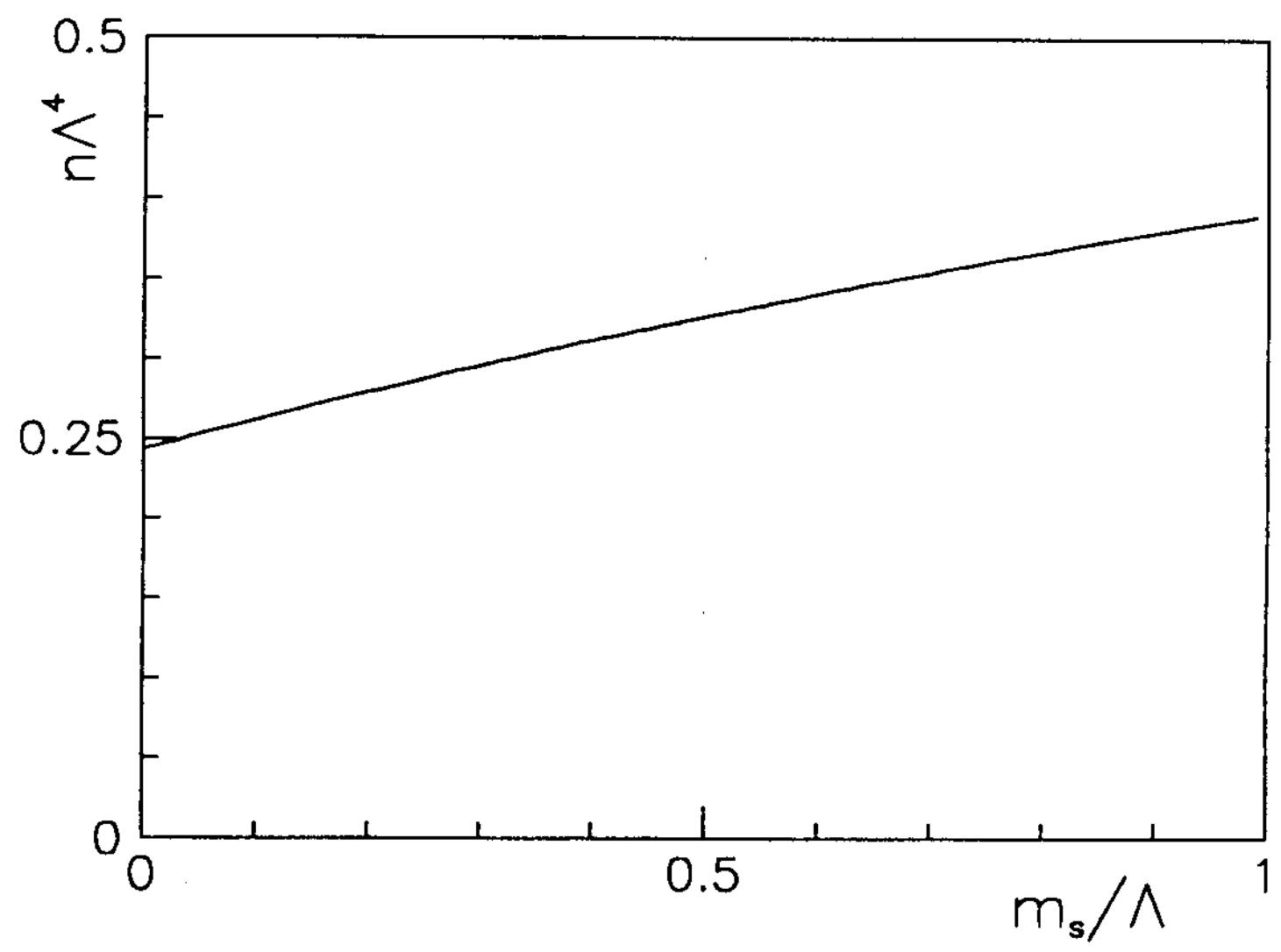

Fig. 1 


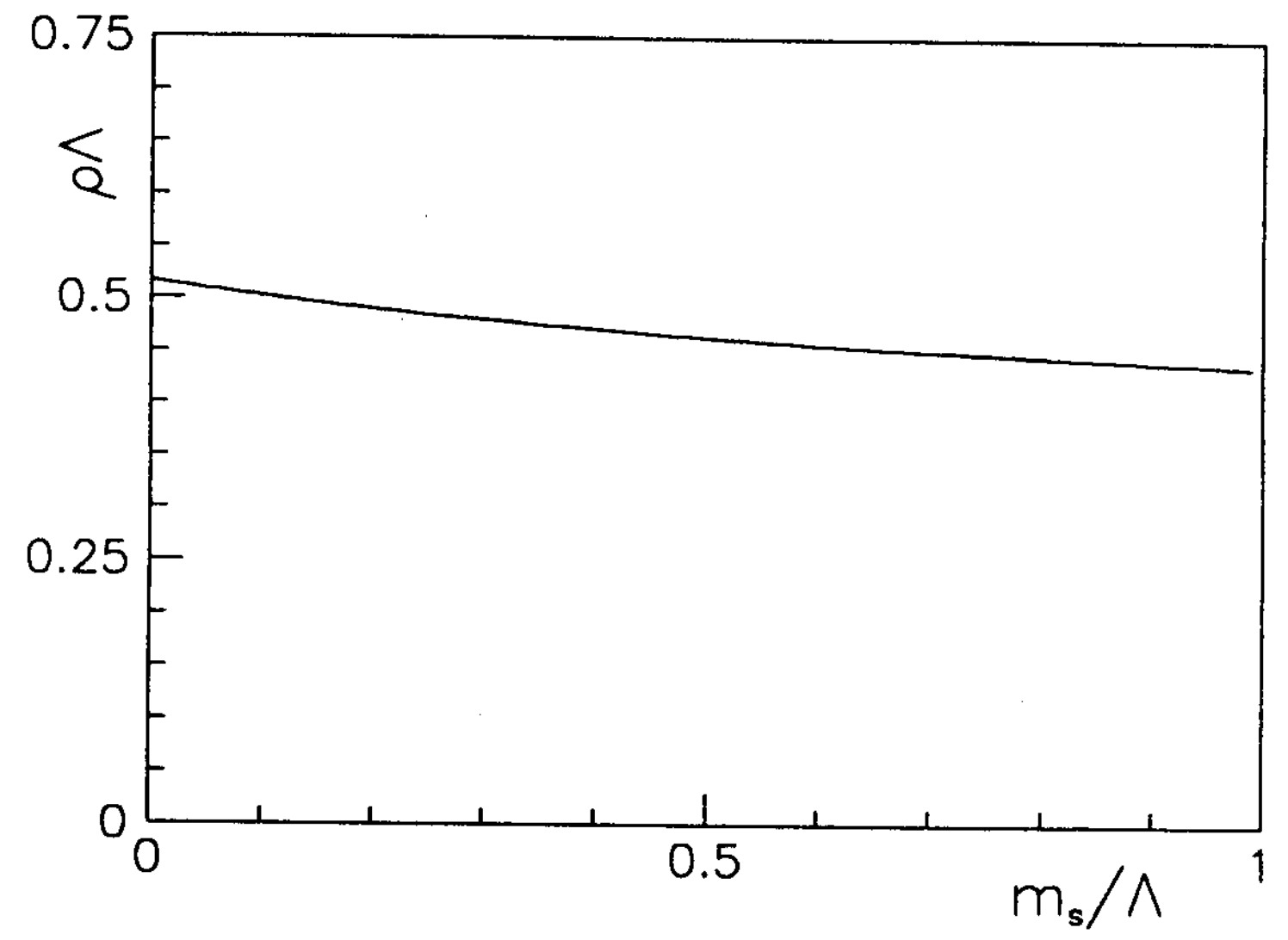

Fig. 2 